\title{
Looking at Prion Diseases in situ with Infrared Microscopy
}

\author{
J. Dubois*, R. Baydack**, and M.Jackson* \\ * Institute for Biodiagnostics, National Research Council Canada, 435 Ellice Ave., Winnipeg, MB \\ R3B 1 Y 6 \\ **University of Manitoba, Department of Medical Microbiology, 750 Bannatyne Ave., Winnipeg, \\ MB R3E 0W3
}

Years of research on the etiology and pathogenesis of diseases have linked many degenerative diseases to aggregated forms of physiological proteins. From the infamous amyloid plaques characteristic of Alzheimer's disease to the mysterious causes of 'protein-only' (prion) diseases such as mad cow and v-CJD, difference are seen in the incubation time, clinical manifestation and even organs involved, but one feature is shared: a normal protein is present in an aggregated form. The Transmissible Spongiform Encephalopathies (TSEs) are a group of invariably fatal neurodegenerative diseases that affect humans and animals. The group includes Creutzfeld-Jakob disease (CJD) and its new variant (nvCJD) in humans, Bovine Spongiform Encephalopathy (BSE) in cattle, and scrapie in sheep. TSEs are characterised by an incubation period of variable duration depending on the strain of prion and species it affects. Rodent models of scrapie have been developed with the advantage of showing short incubation and duration of disease, providing wellsuited model for experimental work.

Experimental scrapie was studied by Fourier Transform Infrared (FTIR) microspectroscopy in two rodent models in order to investigate the chemical differences in the brains of diseased and normal animals. In addition, the spectral information was used to develop a classification algorithm aimed at diagnosing diseased brain sections. The areas of the brains investigated by FTIR were selected based on Magnetic Resonance Imaging (MRI). Information of this nature could provide another means of TSE diagnosis, and it could provide further insight into pathology of prion-associated neurodegeneration.

\section{Experimental protocol}

C57/B1 mice and Syrian golden hamsters were injected intracerebrally with the ME7 and the 263K strains of scrapie, respectively. The presence of lesions in the brains of live hamsters was confirmed by magnetic resonance imaging and by immunostaining in histological slices post-mortem. Hamsters were sacrificed at 49 or 63 days post injection. Mice were sacrificed when they began to show symptoms of disease, at approximately 168 days post injection.

Point infrared microscopy and microscopic imaging were performed using a Bruker IR-Scope II infrared microscope coupled to an IFS 66 infrared spectrometer on 10 micron frozen sections of mouse and hamster brains from control (uninjected), sham (phosphate buffered saline-injected) and prion infected animals.

A classifier was developed using a set of 400 spectra randomly selected from six thousand spectra acquired from mice brains. Six regions were found most suitable for classification and used to develop the linear discriminant analysis.

Results and Discussion 
The infrared spectrum of brain matter is characterised by absorptions from the amide I band and $\mathrm{N}-\mathrm{H}$ bending and $\mathrm{C}-\mathrm{N}$ stretching making up the amide II band (proteins), ring vibrations of carbohydrates, phosphate bonds from nucleic acids, $>\mathrm{C}-\mathrm{O}$ - stretch of ester carbonyls and $\mathrm{C}-\mathrm{H}$ stretching vibrations of $>\mathrm{CH} 2,>\mathrm{CH} 3$ (lipids), all components of typical biological tissues.

Differences in the spectra of grey and white matter are well established [1]

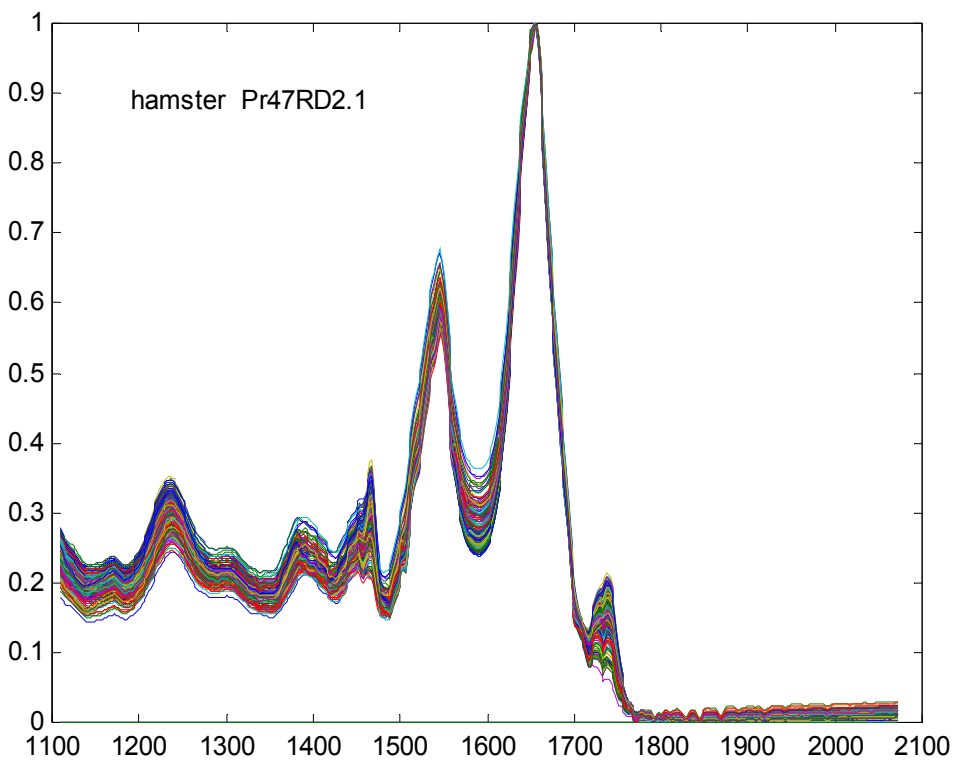

Figure 1: Infrared spectra of hamster brain infected with

and, as expected, these features were present in the spectral maps. Plotting ratios of various infrared absorptions (e.g. lipids on proteins) provides clear pictures of the distributions of certain brain structures, but no clear indication of the presence of prions. One may have suspected the presence of shoulder or even a shift of the amide I band to $1631 \mathrm{~cm}^{-1}$, a position associated with aggregated proteins [2]. However, prion diseases are only seldom characterized by amyloid deposits and $\operatorname{PrP}^{\mathrm{sc}}$ is rather found very dilute in the tissues. Consequently, we and others have not been able to produce spectra with the characteristic feature, even using the higher spacial resolution provided by a focal plane array detector.

In an attempt to make use of the fine spectral differences present between diseased and healthy rodent brain, a classifier based on linear discriminant analysis of selected spectral regions was developed. The classifier used six spectral regions of infrared spectra of mice brains, most of the information being extracted from the amide bands and the fingerprint region. Prediction of the presence of disease was accurate $96 \%$ of the time when applied to test spectra unseen during training or validation. In this presentation, the infrared microscopic picture of brain samples from prion infected animals will be presented in relation with the histology and immunohistology of the samples. Issues relating to investigation of infectious agents with FTIR microscopy will also be discussed.

References :

[1] D.L.Wetzel and S.M. LeVine, Spectroscopy 7 (1993) 40-45.

[2] L.-P. Choo et al., Biophys. J. 71 (1996) 1672-1679.

[4] This research was done in collaboration with Health Canada. The aid of Dr. Tim Booth of the National Microbiology Laboratory and Dr. R. Somorjai and Mrs Saro Bascaramurty from IBD is gratefully acknowledged. 